\title{
Langzeitverlauf eines Patienten mit Mycosis fungoides über mehr als 20 Jahre
}

\author{
A. Koch \\ E. Köstler \\ U. Wollina
}

\author{
Longterm Follow-Up of a Patient with Mycosis Fungoides Over More \\ than 20 Years
}

\section{Zusammenfassung}

Die Behandlung der Mycosis fungoides hat in den letzten Jahren Fortschritte gemacht. Eine Heilung ist dennoch nicht möglich. Die Behandlung muss deshalb zwei wesentliche Ziele verfolgen: Die Kontrolle der Erkrankung, um einen Progress zu verhindern, sowie den Erhalt der Lebensqualität. Langzeitbetrachtungen von Krankheitsverläufen von Patienten mit Lymphomen sind selten beschrieben worden. Wir berichten über einen Patienten mit 20-jährigem Verlauf der Mycosis fungoides, die durch Kombination von topischer und UV-Therapie mit der Röntgenweichstrahltherapie einzelner Herde effektiv kontrolliert werden konnte.

\section{Einleitung}

Die Mycosis fungoides (M. f.) macht in ihrer Gesamtheit 80-85\% aller primär kutanen T-Zell-Lymphome aus [1]. Sie ist eine lymphoproliferative Erkrankung mit Epidermotropie neoplastischer T-Zellen. Wie die Histologie erkennen lässt, ist die begleitende T-Zellreaktion lange Zeit in der Lage, die Expansion der malignen Klone zu begrenzen.

Die 5-Jahres-Überlebensrate aller Mf-Patienten liegt bei 87\% [7]. In den fortgeschrittenen Stadien ist die Prognose ernster mit einem mittleren Überleben von 40 Monaten [1,3,6]. Eine Heilung der M.f. ist bis dato nicht möglich, so dass der Kontrolle der Erkrankung und der möglichen Verhinderung einer Progression die größte Bedeutung im Behandlungskonzept zukommt. Als weiterer wichtiger Aspekt ist der Erhalt der Lebensqualität anzusehen [1].

\section{Fallbericht}

Wir berichten über einen nunmehr 71-jährigen Patienten, der im Herbst des Jahres 1983 erstmals Hautveränderungen im Bereich der rechten Augenbraue bemerkte und sich daraufhin seiner Hausärztin vorstellte. Diese führte unter dem Verdacht einer epidermalen Zyste eine Exzision durch. Der Histopathologe stellte die Verdachtsdiagnose eines Lymphoms. Nach vollständiger Entfernung des auffälligen Herdes in unserer Klinik konnte die Diagnose eines kutanen T-Zell-Lymphoms im Sinne einer Mycosis fungoides mit Mucinosis follicularis bestätigt werden. Im weite- 


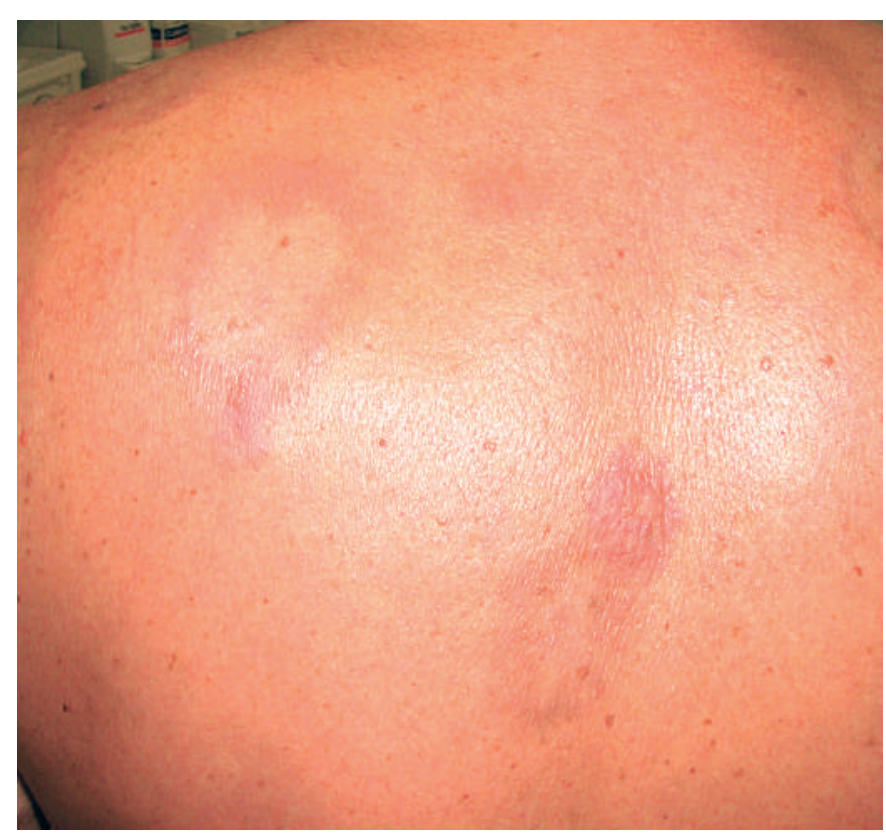

Abb. 1 Plaque und tumoröser Herd einer Mycosis fungoides auf dem Rücken (Verlaufsbild nach 21 Jahren Krankheitsaktivität, 1 - 2005).

ren Verlauf entwickelten sich wiederholt kutane Infiltrate, die als Patches und Plaques sowie als Tumoren imponierten (Abb.1). In den Jahren 1988 und 1992 wurden Basalzellkarzinome an der Nase operativ entfernt.

\section{Histologie}

Augenbraue (1983): regelrechte Parakeratose, Follikelöffnungshyperkeratose, perivasales, vorwiegend aber um Hautanhangsgebilde lokalisiertes, vorwiegend lymphoidzellig differenziertes Infiltrat mit mehrfachem Nachweis von Eosinophilen und mit Eindringen von Infiltratzellen in das subkutane Fettgewebe und in die Wand von Haarfollikeln. Diese zeigen den sich anbahnenden Befund einer so genannten Mucinosis follicularis. Einige Infiltratzellen dringen in die Epidermis ein, hier gelegentlich unter Ausbildung so genannter Pautrierscher Abszesse. Diagnose: Offenbar symptomatische Mucinosis follicularis bei Verdacht auf ein kutanes Lymphom.

Arm (2002): Bei stark unregelmäßig verbreiterter Epidermis mit spongiotischer Lockerung einerseits und ausgedehnten reichlich neutrophilen Granulozyten enthaltenen oberflächlichen Serokrusten findet sich im gesamten überschaubaren Korium und auf das subkutane Fettgewebe übergreifend der Befund eines relativ gemischtzelligen Infiltrats. Es imponieren relativ kleinkernige lymphoide Zellen, massenhaft Plasmazellen, Eosinophile und teilweise neutrophile Granulozyten. Immunhistochemisch stellt sich etwa ein Drittel der Gesamtpopulation als CD3-positive T-Zellen dar mit einer Reihe großkerniger atypischer blastärer Zellen. Etwa ein Drittel der CD3-positiven Zellen ist CD8-positiv. B-Zellen werden in reichlicher Menge zum einen in Form der bereits lichtoptisch gut abgrenzbaren Plasmazellen gesehen, darüber hinaus auch in nicht plasmazellulären T-Zellaggregaten. Umschrieben findet sich eine kleine Ansammlung hoch proliferativer Zellen in subkutanen Infiltratanteilen, der mit an Sicherheit

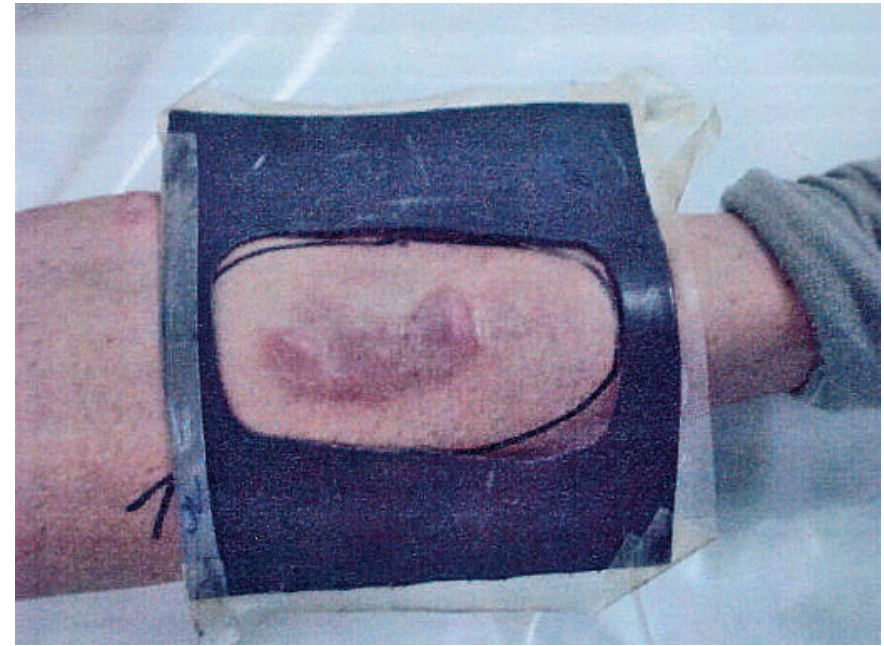

Abb. 2 Röntgenweichstrahltherapie bei Mycosis fungoides mit DARPAC-150, linkes Bein, Feld 112, 4-2003.

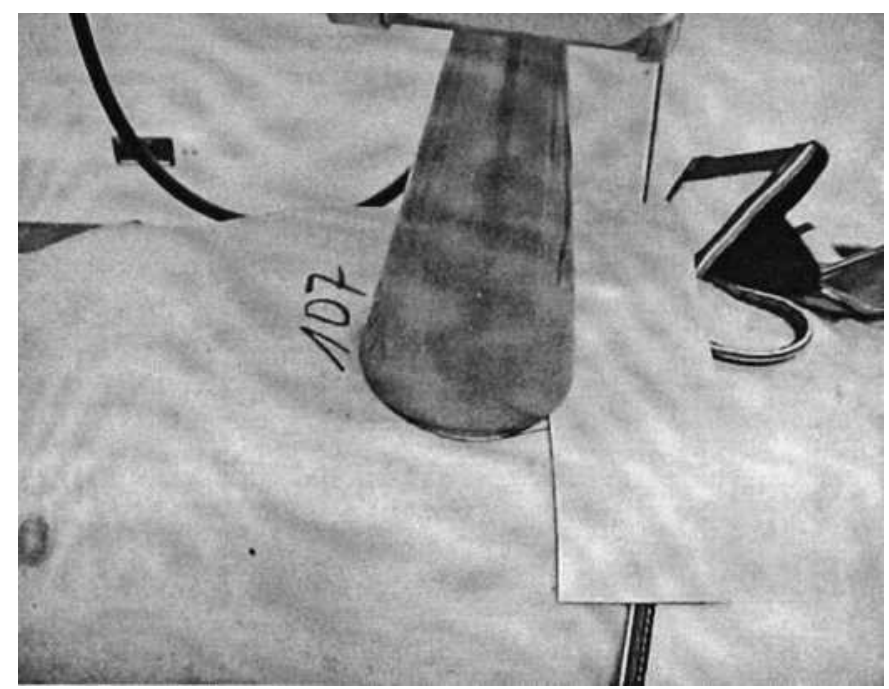

Abb. 3 DARPAC-150 Röntgenbestrahlungsgerät, Tubus $10 \mathrm{~cm}$, oberes Abdomen, Feld 107, 12-2002.

grenzender Wahrscheinlichkeit einem kleinen Keimzentrum zuzuordnen ist. Einzelne großkernige atypische lymphoide Zellen erweisen sich als CD30-positiv. Diagnose: tumorähnliches Plaquestadium der Mycosis fungoides. Die starke Beimengung von Eosinophilen einerseits und Plasmazellen andererseits spricht nicht gegen diese Einordnung, da besonders in ausgedehnten Plaque- und Tumorstadien der M.f. immer wieder diese ungewöhnliche Zellkombination zu sehen ist.

Labor: Sowohl zum Zeitpunkt der Diagnosestellung im Jahre 1983 als auch bei wiederholten Kontrollen konnten keine pathologischen Veränderungen festgestellt werden.

Röntgen-Thorax: Unauffällig.

Diagnose: Mycosis fungoides mit Mucinosis follicularis

Therapie: Mit Ausbildung der ersten großen Plaques und Tumoren im Jahre 1984 erfolgte eine intensive Lokaltherapie mit Clo- 
Tab. 1 EORTC-Klassifikation für primär kutane T-Zell-Lymphome (CTCL) (Willemze et al. 1997)

\begin{tabular}{|ll}
\hline \multicolumn{1}{|l}{ Verlauf } & Histologische Entität \\
\hline indolent & $\begin{array}{l}\text { Mycosis fungoides } \\
\text { Mycosis fungoides mit Mucinosis follicularis } \\
\text { pagetoide Retikulose } \\
\text { großzelliges CD30+ T-Zell-Lymphom } \\
\text { Lymphomatoide Papulose }\end{array}$ \\
\hline aggressiv & $\begin{array}{l}\text { Sezary-Syndrom } \\
\text { großzelliges CD30-T-Zell-Lymphom } \\
\text { provisorisch }\end{array}$ \\
& $\begin{array}{l}\text { Granulomatous slack skin } \\
\text { klein- bis mittelgroßzelliges pleomorphes T-Zell- } \\
\text { Lymphom } \\
\text { subkutanes panniculitis like Lymphom }\end{array}$ \\
\hline
\end{tabular}

Tab. 2 Stadien der Mycosis fungoides

Stadium I (prämykotisch): uncharakteristische Hautveränderungen ähnlich einer Schuppenflechte

Stadium II (infiltrativ): flach erhabene bis kleinknotige Tumoren in der Haut

Stadium III (tumorös): tomatenähnliche oder pilzförmige Tumoren der Haut mit Geschwürbildung

Stadium IV (generalisiert): zusätzlich Befall von Lymphknoten und inneren Organen

betasol, z.T. unter Okklusion sowie eine intermittierende UVTherapie, in den ersten Jahren der Behandlung mit UVB, im weiteren Verlauf Creme-PUVA. Die UV-Therapie erfolgte vorwiegend in den Herbst- und Wintermonaten, wobei im Sommer die natürliche Sonnenstrahlung genutzt wurde.

Für stark infiltrierte Plaques und tumoröse Herde wurde eine lokale Röntgenweichstrahltherapie eingesetzt. Dabei wurden bisher 120 Felder mit Gesamtdosen pro Feld zwischen 6 und 15 Gray bei 30 bis $80 \mathrm{kV}$ bestrahlt. Die Einzeldosis lag in der Regel bei 1,5 Gray (Abb. 2 und 3). Einzelne Herde wurden exzidiert.

Ergebnis: Bisher wurde kein Progress der M.f. festgestellt (1983: Stadium IIb; 2003: Stadium IIb).

\section{Diskussion}

Patienten mit einem kutanen T-Zell-Lymphom (Tab.1) haben häufig bereits Jahre vor der Diagnosestellung erste Hautveränderungen bemerkt. Die Befunde in der frühen Krankheitsphase sind häufig nicht spezifisch und weisen bei der Biopsie keine typi- schen histologischen Veränderungen auf. Mittels T-Zell-Rezeptor-Rearrangement lassen sich diese Infiltrate jedoch als klonale T-Zell-Proliferate identifizieren [3].

Die M. f. verlangt nach einer stadiengerechten Therapie (Tab. 2) [7]. Patienten im Stadium Ia haben eine Lebenserwartung, die der einer alters- und geschlechtsbezogenen Vergleichsgruppe entspricht. Die mediane Überlebenszeit beträgt für Patienten mit ausgedehnten Patches oder Plaques (Stadium Ib oder Ila) 11 Jahre. Patienten, die im Stadium IIb der Erkrankung mit kutanen Tumoren auffallen haben eine mediane Lebenszeit von 3,2 Jahren. Bei Patienten mit Erythrodermie (Stadium III) sowie mit extrakutaner Manifestation im Stadium IVa oder IVb besteht eine mittlere Überlebenszeit von 4,6 Jahren (Erythrodermie) respektive 1,2 Jahren (Stadium IVa) und 0,9 Jahren beim Stadium IVb [4].

Unser Patient befand sich zum Zeitpunkt der Diagnosestellung in einem Stadium IIb. Durch die stadiengerechte Behandlung mit topischen Kortikosteroiden, UV-Therapie sowie Exzisionen und Röntgenweichstrahlentherapie konnte der weitere Progress verhindert werden. Die mittlere Überlebenswahrscheinlichkeit für unseren Patienten lag nach den oben angeführten statistischen Angaben bei 3,2 Jahren. Der Krankheitsverlauf spricht allerdings gegen therapeutischen Nihilismus.

Die Weichstrahlröntgentherapie, welche seit Jahrzehnten für die M. f. verwendet wird, hat nach wie vor ihre Berechtigung [2]. Ihr Vorteil ist die Gewebeverträglichkeit durch Fraktionierung und Schonung der gesunden Haut.

\section{Literatur}

${ }^{1}$ Bunn PA Jr, Hoffmann SJ, Norris D, Golitz LE, Aeling JL. Systemic therapy of cutaneous T-cell lymphomas (mycosis fungoides and the Sezary syndrome). Ann Intern Med 1994; 121: 592-602

${ }^{2}$ Chaoul H, Wachsmann F. Allgemeines über die Anwendungsgebiete der Nahbestrahlung und der erweiterten Nahbestrahlung. Die Nahbestrahlung. Stuttgart: G. Thieme Verlag, 1953

${ }^{3}$ Diamandidou E, Cohen PR, Kurzrock R. Mycosis fungoides and Sezary syndrome. Blood 1996; 88: 2385 - 2409

${ }^{4}$ Koh HK, Charif M, Weinstock MA. Epidemiology and clinical manifestations of cutaneous T-cell lymphoma. Hematol Oncol Clin North Am 1995; 9: $943-960$

${ }^{5}$ Smith BD, Wilson LD. Management of mycosis fungoides. Part 1. Diagnosis, staging, and prognosis. Oncology 2003; 17: 1281-1288

${ }^{6}$ Van Doorn R, Van Haselen CW, van Voorst Vader PC et al. Mycosis fungoides: disease evolution and prognosis of 309 Dutch patients. Arch Dermatol 2000; 136: 504-510

${ }^{7}$ Willemze R, Kerl H, Sterry W et al. EORTC classification for primary cutaneous lymphomas. A proposal from the Cutaneous Lymphoma Study Group of the European Oganization for Research and Treatment of Cancer. Blood 1997; 90: 354-371 